\title{
NEAR DEFECT FREE GaN SUBSTRATES
}

\author{
S. POROWSKI \\ High Pressure Research Center, Polish Academy of Sciences, \\ Sokolowska 29/37, 01-142 Warsaw, Poland, sylvek@ unipress.waw.pl
}

The current status of GaN crystallization under high nitrogen pressure will be presented. Both conductive and semi-insulating GaN crystals will be characterized.

In particular the influence of $\mathrm{Mg}$ on the growth mechanisms will be discussed. The influence of $\mathrm{Mg}$ doping on morphology of $\mathrm{Mg}$-doped crystals grown under pressure and $\mathrm{Mg}$ doped homoepitaxial layers will be shown. It will be also shown that the addition of about 1 at.\% of $\mathrm{Mg}$ into the solution improves significantly the structural quality of crystals reducing dislocation density at least by 3 orders of magnitude comparing to the crystals grown without an intentional doping. As it was estimated by selective wet etching and transmission electron microscopy the dislocation densities in the $\mathrm{Mg}$-doped $\mathrm{GaN}$ is as low as $10 \mathrm{~cm}^{-1}$. The introduction of $\mathrm{Mg}$ also lowers the optical absorption coefficients for energies below fundamental edge by 2 to 3 orders of magnitude what is explained by disappearance of defect related states in the gap.

The procedures for preparation of atomically flat epi-ready (000 1 ) surfaces without subsurface damage will be described. It will be shown that high quality homoepitaxial layers growing by monoatomic steps are possible on these substrates.

\section{INTRODUCTION}

The technology of GaN and its ternaries, GaAlN and GaInN is of crucial importance for blue optoelectronic devices. Progress in technology and basic research of GaN led to development of blue [1], green and yellow [2] light emitting diodes (LEDs), which are now commercially available from a number of sources. Also the low power laser diodes have been announced [3]. Still the stability and power of laser diodes are not satisfactory.

These results have been achieved using either sapphire or SiC substrates. Further progress can be attained by either developing more sophisticated layer growth techniques such as epitaxial lateral overgrowth (ELOG) or by developing GaN substrates. Essentially both methods rely on the same phenomenon, i.e. enforcing fast growth along (1010) direction in order to obtain large area substrate having reduced dislocation density. Due to absence of destabilizing factor of contact with the foreign substrate, the growth of single crystals is more promising technique.

Growth of GaN single crystals have been subject of the research for several years [4,5]. Using High Pressure Solution Growth (HPSG) method $100 \mathrm{~mm}^{2}$ size GaN plate-like single crystals of good crystallographic quality have been obtained. Both n-type and semiinsulating (SI) crystals have been grown [6]. Also GaN surface preparation techniques have been developed, which include mechano-chemical polishing for active ( $\mathrm{N}$ face) surface [7] of GaN platelets and Reactive Ion Etching (RIE) for inert (Ga face) surface.

GaN single crystal substrates were successfully used for MBE and MOCVD epitaxial growth. Homoepitaxial layers of very good crystallographic quality, resulting from good quality of the substrate and undisturbed epitaxial growth by flow of monoatomic steps. Recently, the dislocation-free multiquantum well (MQW) structures have been obtained.

In this paper we will discuss recent development of the growth of $\mathrm{GaN}$ single crystals. First the thermodynamic conditions for the growth of GaN crystals from the constituents: liquid $\mathrm{Ga}$ and $\mathrm{N}_{2}$ under high pressure will be obtained. Then the physical properties, including crystallographic, optical and electric will be discussed. Subsequently the results of surface 
preparation techniques to GaN single crystals will be illustrated by results of microscopic and structural investigations. Finally the results of epitaxy both by MOCVD and MBE will be discussed.

\section{PHYSICAL PROPERTIES OF Ga(l)-N2-GaN SYSTEM AND GaN CRYSTAL GROWTH}

Thermodynamic and kinetic properties of $\mathrm{Ga}(\mathrm{l})-\mathrm{N}_{2}-\mathrm{GaN}(\mathrm{s})$ system are determined mostly by strong bonding both in $\mathrm{N}_{2}$ molecule and in $\mathrm{GaN}$ crystal. The transition between the initial state of liquid gallium and nitrogen $\mathrm{N}_{2}$ and $\mathrm{GaN}$ crystal requires breaking the extremely strong bonding in $\mathrm{N}_{2}$ molecule (bond energy $9.8 \mathrm{eV} /$ molecule) and creation of $\mathrm{GaN}$ bonds. Strong bonding in GaN crystal (bond energy (9.32 eV/atom pair) leads to high melting temperature $\mathrm{T}^{\mathrm{M}}{ }_{\mathrm{GaN}} \sim 2800 \mathrm{~K}$ [8]. The estimated $\mathrm{N}_{2}$ equilibrium pressure for $\mathrm{GaN}$ is over 45

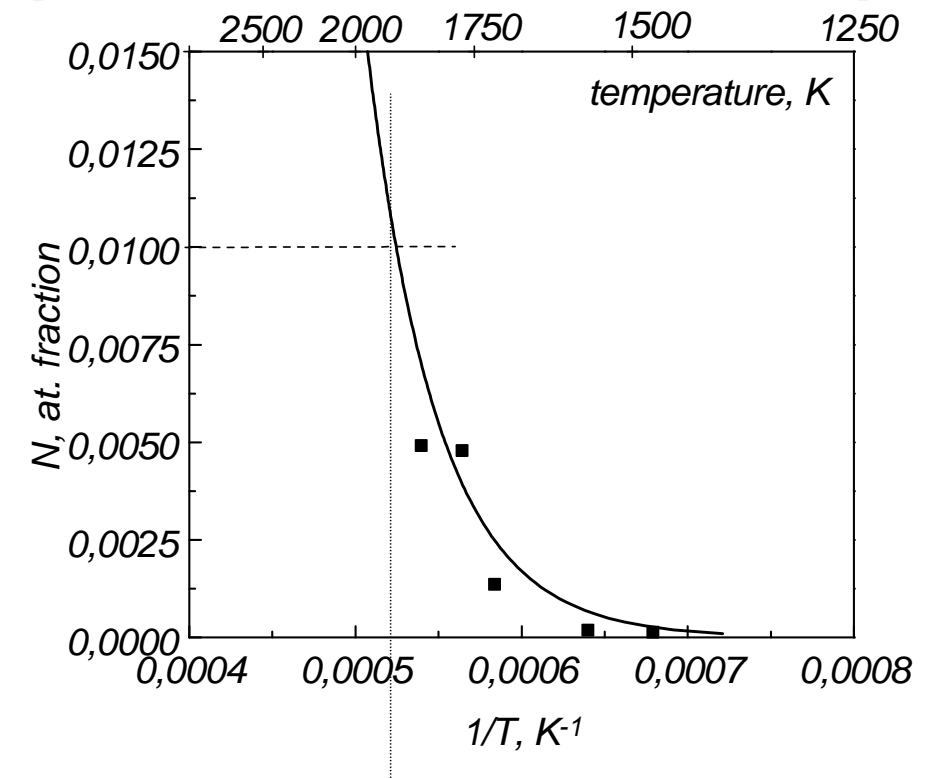

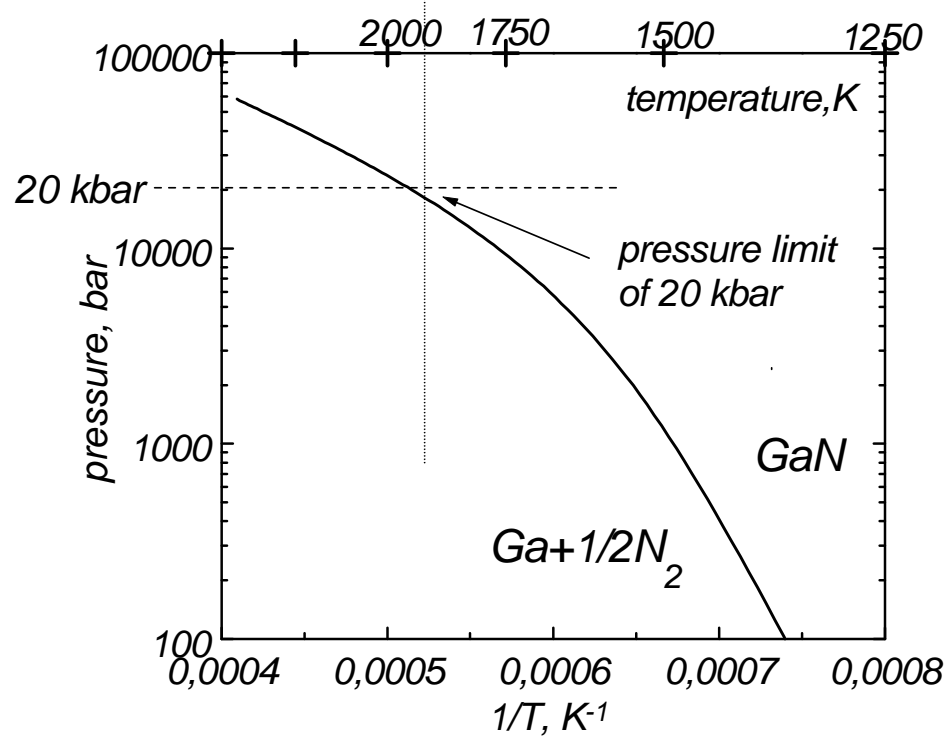

Fig 1. $\mathrm{N}_{2}(\mathrm{~g})$ - $\mathrm{Ga}(\mathrm{l})$ - $\mathrm{GaN}(\mathrm{s})$ phase diagram: a - p-T coordinates [9]; b - x-T coordinates [10] kbar corresponds to nitrogen solubility in liquid gallium of the order of $1 \%$. Lower temperatures correspond to lower nitrogen solubility, therefore by controlling the temperature 
difference in the crucible we can control the difference of the nitrogen concentration and accordingly the supersaturation in the growth zone. Our growth results suggest that the nitrogen solubility and diffusion from the hot to cold zone of the gallium is the rate limiting step in the growth.

Due to relatively rapid dissolution of nitrogen in liquid $\mathrm{Ga}$, in the beginning of the process $\mathrm{GaN}$ polycrystalline crust is created on the Ga surface. Some of the crust GaN crystals grow faster and inhibit the growth of neighboring crystals. Depending on the supersaturation and the temperature the two growth habits are obtained: needle-like habit which dominates for large supersaturation (large temperature difference ) and plate-like habit which is prevalent for lower supersaturations (small temperature gradient in the crucible).

It has been found that morphology and quality of the GaN crystals depend crucially on the doping of growth solution. The morphology of the two principal surfaces of GaN platelets is different: one side is completely flat and the other is corrugated, with large number of multi-atomic steps, ridges and even growth hillocks. For undoped Ga solution the corrugated surface correspond to Ga face ((0001) face) and the flat surface to $\mathrm{N}$ face $(0001)[12,13]$. The addition of $\mathrm{Mg}$ to liquid $\mathrm{Ga}$ changes the morphology of the plate - Ga face becomes completely flat and $\mathrm{N}$ face is corrugated [6]. Also the electric and optical properties are different - Mg doped crystal have very low concentration of free carriers and at room temperature these crystals are semiinsulating [6].
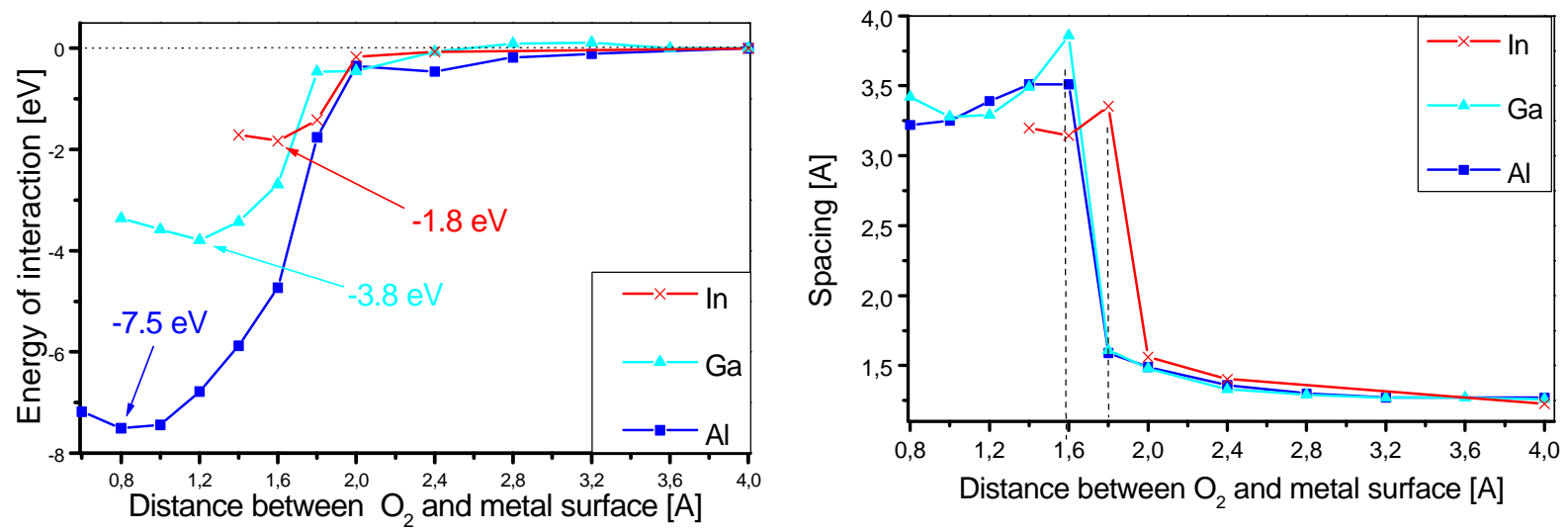

Fig. 2. Interaction between $\mathrm{O}_{2}$ molecule and metal III group surface: a - excess energy, b - O - O distance in oxygen molecule dependence on the distance between the molecule and the metal surface.

High concentration of free electrons in $\mathrm{GaN}$ grown from undoped $\mathrm{Ga}$ is attributed either to nitrogen vacancies [14] or to oxygen substitutional impurity [15]. The incorporation of oxygen is related to its dissolution in liquid metal. This process has been investigated using quantum mechanical calculations. The results have been obtained using commercially available Dmol package distributed by Molecular Simulation Inc. within countrywide license in Poland [16] results of these calculations have been shown in Figure 2. In Figure 2a the excess energy of the oxygen molecule approaching the liquid metal group III surface is shown. As it can be seen, the adsorption of oxygen on liquid metal surface is barrierless process. This type of the adsorption energy curve is standard for the molecular adsorption. The nature of oxygen adsorption is different - the $\mathrm{O}_{2}$ molecule disintegrates on the surface. On Figure $2 \mathrm{~b}$ the spacing between the oxygen atoms is presented as a function of the distance between the molecule and the metal surface. As it can be seen the distance between the oxygen atoms rapidly increases from 1.53 to above $3 \AA$. This proves that adsorption leads to dissociation of oxygen molecule on the metal surface. Moreover, the barrierless character of the process indicates that the incorporation of oxygen is very efficient and that liquid gallium absorbs all oxygen from gaseous nitrogen. 

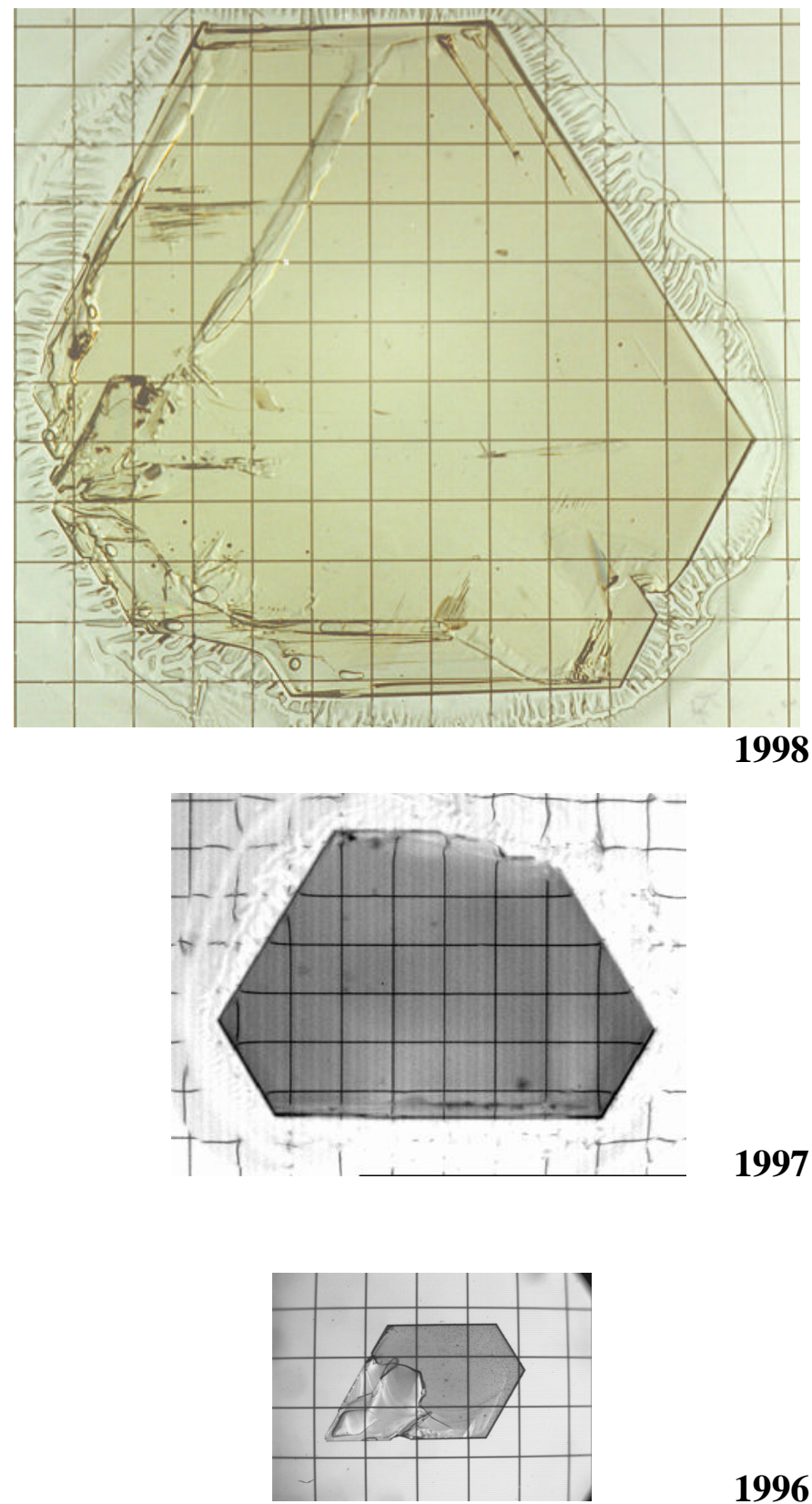

1998

1996

The main problem in growth of high quality crystals is how to attain high growth rate and preserve the morphological stability of the surface. The growth rate can be increased either by higher equilibrium concentration of $\mathrm{N}$ in liquid $\mathrm{Ga}$ or by increase of the supersaturation. In our growth method we keep the supersaturation at the lowest possible level and increase the solubility by increasing the temperature of the liquid gallium. This leads to the increase of the growth rate and reduction of the destabilizing factor of high supersaturation The destabilizing influence of impurities is also reduced by preserving high purity of the process. This is achieved by initial annealing of the high pressure vessel in the temperature of $200^{\circ} \mathrm{C}$ and vacuum of $10^{-3}$ Torr.

The improvement of the growth procedure led to considerable progress of $\mathrm{GaN}$ high pressure growth which is illustrated in Figure 3. The typical size of the undoped GaN crystal, obtained in

$1997200 \mathrm{~h}$ growth process is above $15 \mathrm{~mm}$ in length with the surface area above 100 $\mathrm{mm}^{2}$.

The size of $\mathrm{Mg}$ - doped GaN crystals obtained in the process of the same duration is about $10 \mathrm{~mm}$ with the area of $80 \mathrm{~mm}^{2}$. The size difference is related to reduction of the growth rate in the plane perpendicular to $\mathrm{c}$ axis for Mg-doped GaN crystals.

Fig. 3 Progress in GaN high pressure crystallization - increase of the size of GaN crystals in last 3 years,

\section{PHYSICAL PROPERTIES OF PRESSURE GROWN GaN CRYSTALS}

The HPSG method allows to grow both conductive and non-conductive crystals of GaN. The electrical properties depend on the solvent used for crystallization. As it is summarized in Fig. 4, GaN crystals grown from solutions in pure Ga, without an intentional doping, are highly conductive with Fermi level lying in the conduction band. The growth from solutions in $\mathrm{Ga}$ alloyed with small amount of $\mathrm{Mg}$ results in highly resistive crystals with Fermi level shifted into the gap. Fig. 5 shows temperature dependence of resistivity [17] for both types of crystals. It allows to compare the type of conductivity in undoped and $\mathrm{Mg}$ doped crystals. 


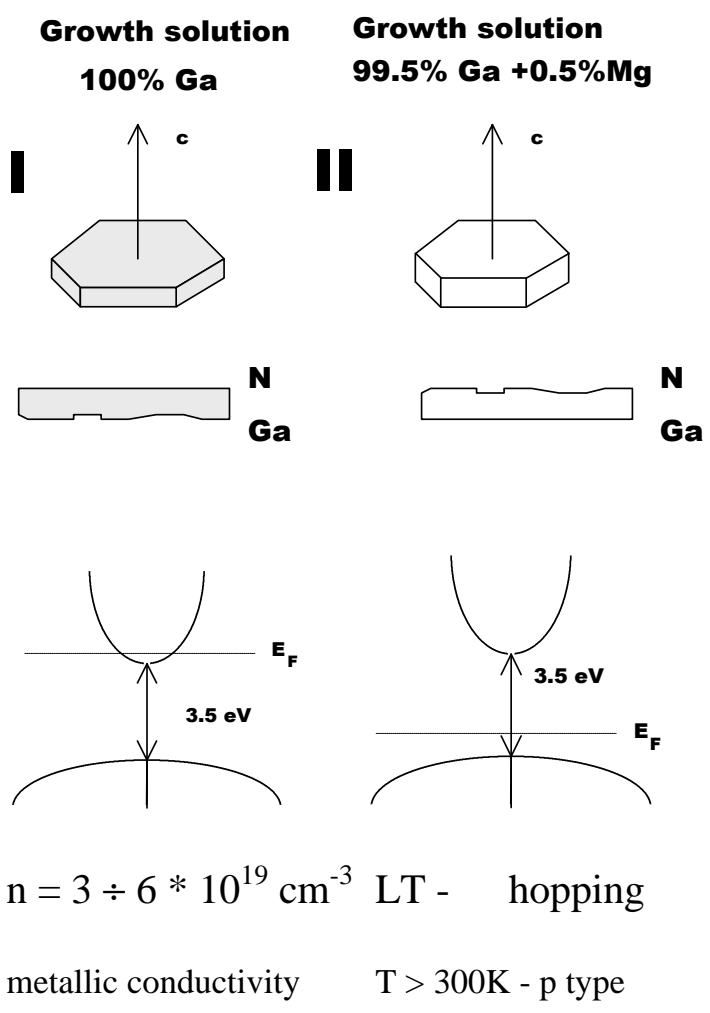

Fig. 4 Electric properties and morphology of GaN crystals: I - undoped, II - Mg - doped.

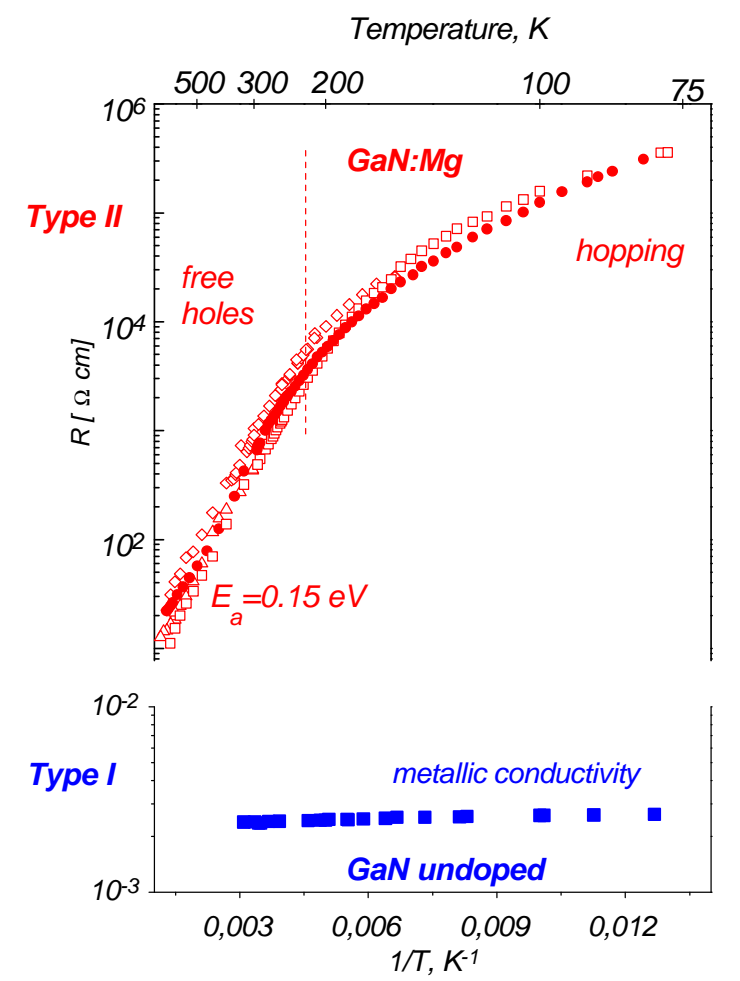

Fig. 5 Temperature dependence of resistivity of undoped and Mg-doped GaN crystals

GaN crystals grown from the solutions in pure gallium show metallic behavior in whole temperature range of $4.2-300 \mathrm{~K}$. High free electron concentration of 3-6 x $10^{19} \mathrm{~cm}^{-3}$ with mobilities of $\left.30-90 \mathrm{~cm}^{2} / \mathrm{Vs}[18,17]\right]$ have been found (Table I). The main residual impurity detected in the crystals by SIMS [19] is oxygen. Its concentration was estimated to be in the range of $10^{18}-10^{19} \mathrm{~cm}^{-3}$. It is well established that oxygen is a single donor in $\mathrm{GaN}$. However since the concentration of free electrons in the crystals is higher than the estimated concentration of the impurity the presence of an additional donor cannot be excluded. The Nvacancy as the additional source of free electrons is therefore often proposed [20,21].

Following the theory [20,22], highly probable native defects in $\mathrm{GaN}$ crystals which are highly n-type, are Ga-vacancies $\left(\mathrm{V}_{\mathrm{Ga}}{ }^{3-}\right)$. This is due to their low formation energy even at strongly Ga-rich conditions of crystallization. The presence of the negatively charged $\mathrm{Ga}$ vacancies at concentrations of $10^{18} \mathrm{~cm}^{-3}$, in the n-type pressure grown GaN crystals, has been detected by positron annihilation experiments [23].

The addition of $\mathrm{Mg}$ into the growth solution drastically changes the electrical properties of GaN crystals. Their RT resistivity increases by orders of magnitude (Table I).

Table I Electrical properties of GaN crystals

\begin{tabular}{llll}
\hline & & $\rho$ & \\
crystal & conductivity type & $\Omega \mathrm{cm}, 300 \mathrm{~K}$ & carrier concentration,cm ${ }^{-3}$ \\
\hline $\mathrm{GaN}$ & metallic & $10^{-3} 10^{-2^{-}}$ & $3-6 \times 10^{19}$, n-type \\
$\mathrm{GaN}: \mathrm{Mg}$ & hopping & $10^{4}-10^{6}$ & ---- \\
\hline
\end{tabular}

The increase of electrical resistance in $\mathrm{GaN}: \mathrm{Mg}$ crystals is related to drastic decrease of free electron concentration. The temperature dependence of resistivity for these samples at low temperatures up to about $250 \mathrm{~K}$, suggests hopping type of conductivity. At higher 
temperatures the conductivity starts to be governed by the activation process leading to the creation of free holes in the valence band.

Also the optical absorption data [24] indicate that the free carrier concentration in the $\mathrm{Mg}$-doped GaN is very low. The free carrier absorption which dominates the low energy part of the absorption spectra for the undoped GaN disappears completely for crystals grown from Mg containing solutions. For these crystals, the absorption coefficients for energies below the fundamental absorption edge are as low as $1-10 \mathrm{~cm}^{-1}$ as measured by Photothermal Deflection Spectroscopy . [25].
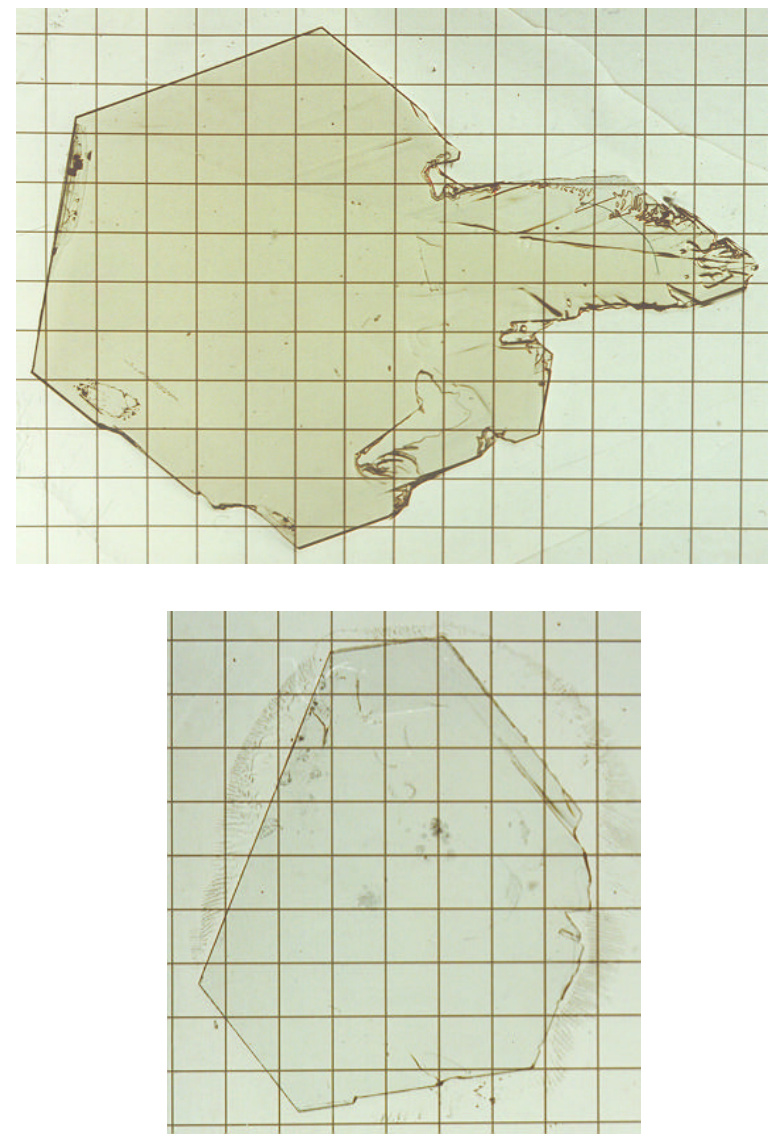

Fig 6 GaN undoped (top) and Mg-doped (bottom) single crystals. (grid spacing - 1mm).
The Mg impurity introduced into the growth solution does not change significantly the general habit of the crystals. In both cases they are hexagonal platelets with c-axis perpendicular to the hexagonal faces. Typical crystals of both types are shown in Fig. 6. The crystals with $\mathrm{Mg}$ are perfectly transparent and colorless whereas the conductive samples are usually slightly yellowish in color.

From the form of the crystals, it follows that the relative growth rates in directions perpendicular and parallel to the caxis are similar however the crystals with $\mathrm{Mg}$ are generally thicker and require longer time to reach the same linear dimensions as the crystals grown without $\mathrm{Mg}$.

One of the hexagonal faces of both types of crystals is usually flat. The opposite one often shows some macro-roughness (i.e. macrosteps) as a consequence of the unstable growth in one of the (0001) directions. As it was revealed [26] by wet etching [7] and confirmed by CBED measurements $[12,13]$, the flat surfaces of the conductive and nonconductive crystals correspond to opposite polar (0001) orientations of GaN.

For conductive GaN crystals the flat surface is always $(0001)_{\mathrm{N}}$ face which is chemically active [7]. For the Mg-doped crystals this is always $(0001)_{\mathrm{Ga}}$ face which is resistant to the wet etching in alkali water solutions.

The GaN crystals grown by HPSG method are of high structural quality as determined by X-ray diffraction (XRD) [27], transmission electron microscopy (TEM) [12, 28] and defect selective wet etching (etch pit density - EPD) techniques. The XRD data are summarized in Fig. 7. The X-ray rocking curves for symmetrical reflection are as narrow as 18-25 arcsec for almost all Mg-doped crystals and for best crystals grown without doping. For some of the conductive crystals the rocking curves splits onto few peaks indicating low angle (about 1 arcmin) grain boundaries. This is probably due to some inhomogeneity in distribution of residual donor impurities. The rocking curves for in-plane reflections are always very narrow what indicates that there is no twist mosaicity in all investigated crystals.

A number of GaN crystals have been studied by TEM [12,13,28] especially the samples used for optimization of surface preparation procedures and epitaxial growth of 
layers and structures. In most observations the crystals were completely free of dislocations. Fig. 8 shows a $3 \mu \mathrm{m}$. TEM cross-sectional view [28] of the GaN conductive crystal with $\mathrm{GaN} / \mathrm{AlGaN}$ structure grown by MBE [29] on the active $(0001)_{\mathrm{N}}$ surface. In this measurement no dislocations have been found over $100 \mu \mathrm{m}$. area. It suggests that the dislocation density in the crystal is not higher than $10^{4} \mathrm{~cm}^{-2}$.

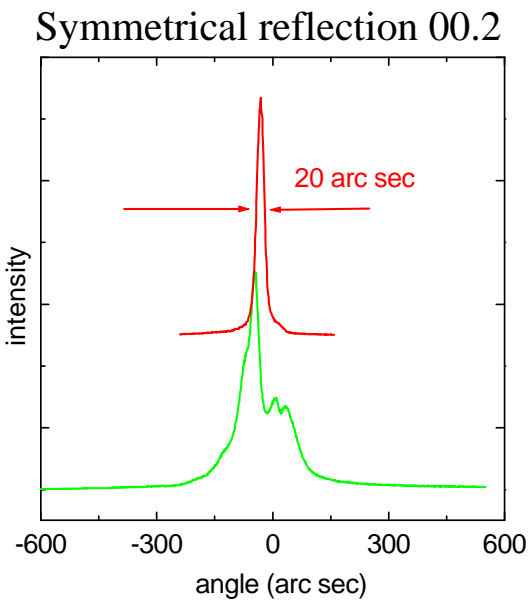

in plane reflection 22.0

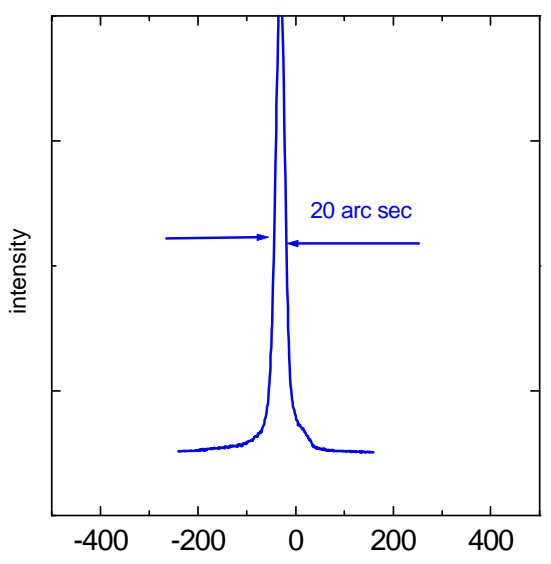

Fig.7 x-ray rocking curves of pressure GaN crystals

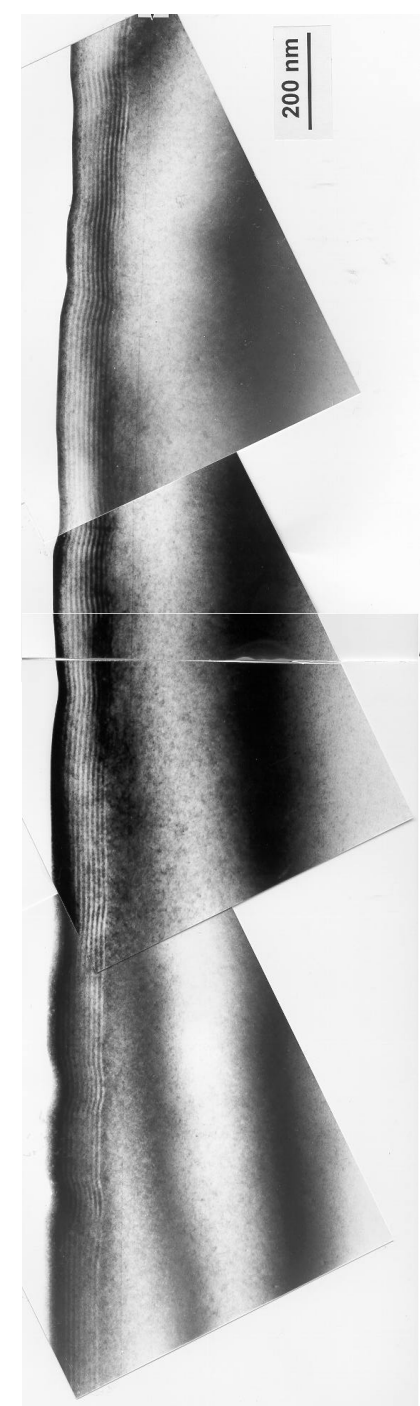

Fig. 8 TEM crossectional view of MQW structure grown on $\mathrm{GaN}$ substrate. Courtesy of M. Albrecht .

It was mentioned earlier that the Ga-side of $\mathrm{GaN}$ cannot be etched in alkali water solutions. However if molten $\mathrm{KOH}, \mathrm{NaOH}$ or their molten eutectic are applied the defect selective etching of GaN heteroepitaxial layers and single crystals is possible. The method developed for $\mathrm{GaN}$ heteroepitaxial layers [7] has been applied to pressure grown GaN crystals doped with $\mathrm{Mg}$. The number of the etch pits on heteroepitaxial GaN corresponded to defect density of $2 \times 10^{8} \mathrm{~cm}^{-2}$ whereas only very few such a pits have been found on the whole surface (few tens of $\mathrm{mm}^{2}$ ) of GaN single crystal.

The estimations for dislocation density in pressure grown $\mathrm{GaN}$ crystals are summarized in Table II. 
Table II Dislocation density in pressure grown GaN crystals

\begin{tabular}{rc}
\hline Type of crystal & Dislocation density, $\mathrm{cm}^{-2}$ \\
\hline GaN, undoped, conductive & $10^{4}$ \\
\hline GaN:Mg, non-conductive & $10^{1}-10^{2}$ \\
\hline
\end{tabular}

\section{SURFACE PREPARATION}

The surfaces of the pressure grown GaN crystals are often covered by the growth figures or surface layer resulting from the cooling of the system after crystallization at high temperature. Therefore to obtain epi-ready surfaces of GaN substrates it is necessary to subject them to the preparation procedures prior to epitaxial growth.

The mechanical polishing with diamond micropowders leads to the formation of highly damaged surfaces with scratches of $200 \AA$ in depth. The thickness of the damaged layer under such mechanically polished surfaces is usually $2000-2500 \AA$ as it was shown by the RBS measurements [30] (Fig. 9).

It was shown that bulk GaN crystals (highly conductive, undoped) can be etched in aqueous solutions $(10 \mathrm{~N}-1 \mathrm{~N})$ of $\mathrm{KOH}$ and $\mathrm{NaOH}$ [7]. However only one of the polar $\{0001\}$ surfaces of bulk crystals is attacked by the applied etchants. The free etching of this surface $(0001)_{\mathrm{N}}$ as it was identified by XPD [13]) is strongly anisotropic and results in the formation of numerous stable pyramids $100-200 \mathrm{~nm}$ in height. As it was mentioned before for $\mathrm{Mg}$ doped crystals also the $(0001)_{\mathrm{N}}$.face is chemically active.

The same aqueous solutions $(10 \mathrm{~N}-1 \mathrm{~N})$ of $\mathrm{KOH}$ and $\mathrm{NaOH}$ have been used for mechano-chemical polishing of the chemically active GaN surface [7]. When the mechanochemical polishing procedure is applied, atomically flat surfaces of bulk GaN (RMS $=0.1 \mathrm{~nm}$ as estimated by AFM) are reproducibly obtained. The RBS measurements [30] (Fig.9) indicate that the applied procedure allows to remove the subsurface damage resulting from the mechanical polishing. It was confirmed by TEM studies of subsurface regions of mechanochemically polished GaN crystals. One of the cross sectional TEM views of such a crystal is shown in Fig. 10.

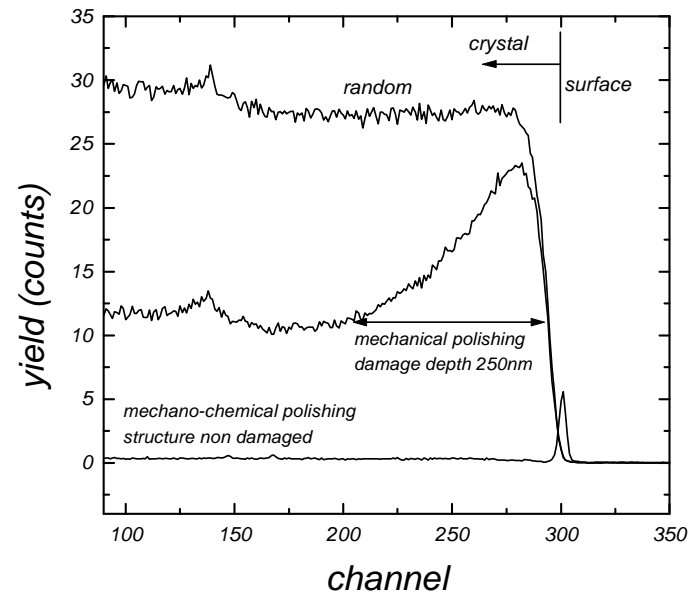

Fig. 9 RBS signals for polished GaN surfaces, Ref. 12

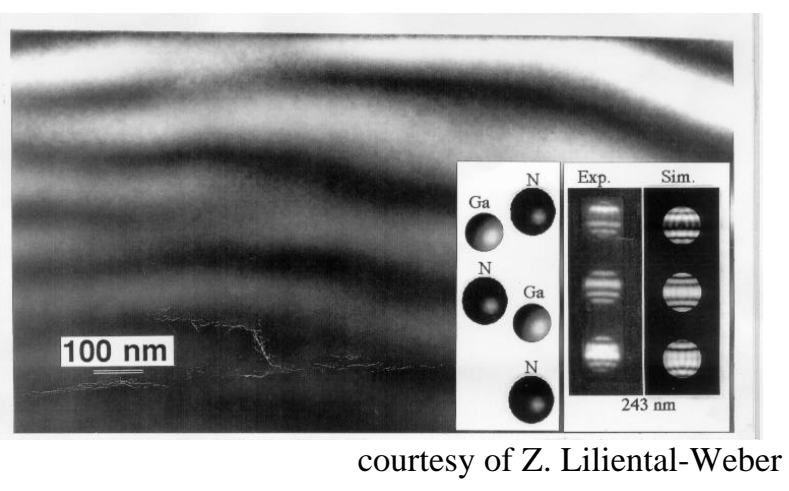

Fig.10 Cross sectional TEM view of GaN crystal at the mechano-chemically polished surface. The insert shows CBED spectra used for polarity determination.

The procedures of the wet etching and mechano-chemical polishing with alkaline water solutions can be also applied for $\mathrm{GaN}$ heteroepitaxial layers but only to that which 
shows tendency to develop hexagonal hillocks if grown by MOCVD. The orientation of this chemically active epitaxial GaN layers is the same as the orientation of chemically active surface of the conductive GaN crystals.

The surfaces of $\mathrm{GaN}$ crystals corresponding to the Ga-polarity faces cannot be prepared by the methods just described due to their chemical resistance to most chemicals, at room temperature. Therefore for these surfaces, the Reactive Ion Etching techniques are used for removing the subsurface damage introduced by the mechanical polishing. The application of RIE allows to remove the subsurface damage what was shown by TEM [28]. Some roughness remaining after the RIE treatment is usually removed at the first stages of epitaxial growth.

\section{HOMOEPITAXY}

Epitaxial growth of GaN layers and GaN-based structure was tried on both polar surfaces of pressure grown GaN crystals, by MOCVD [31, 32, 33, 34,] as well as by MBE with plasma [29] and ammonia [35] nitrogen sources.

The growth by propagation of monoatomic steps has been achieved by both methods on both polar surfaces. Fig. 11 shows the morphology of $\mathrm{GaN}$ homoepitaxial layer grown by $\mathrm{MBE}$ with $\mathrm{NH}_{3}$ nitrogen source $[\mathrm{gn}]$ on the $(0001)_{\mathrm{N}}$ prepared by mechano-chemical polishing.
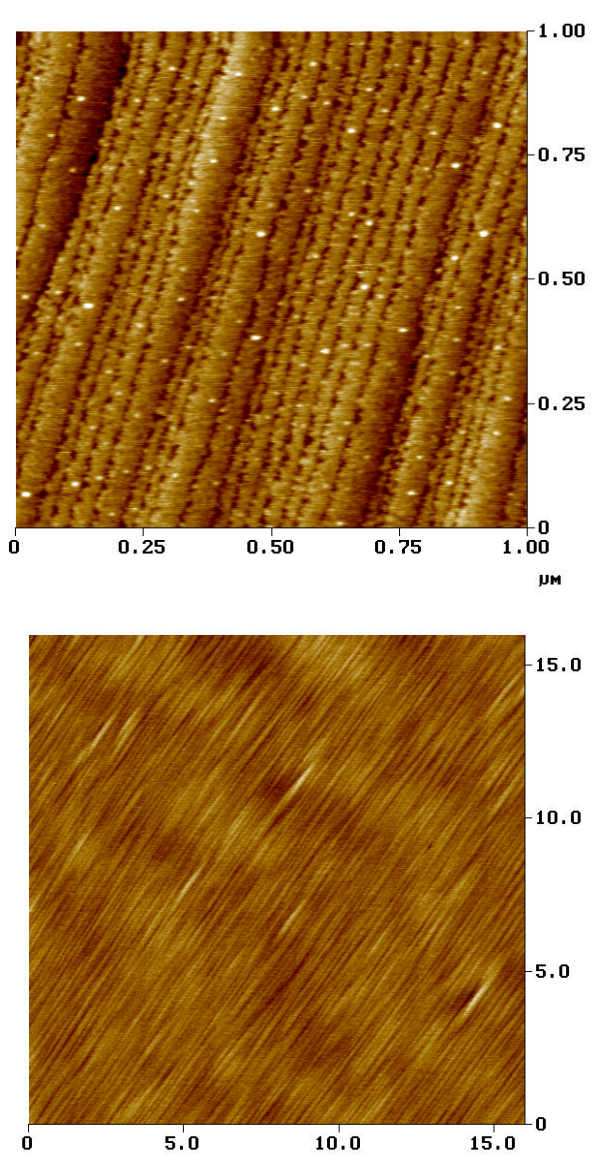

Fig. 11 AFM scans of GaN homoepitaxial layer grown on the active side of GaN crystal by MBE with ammonia N source [35]
Such a sequence of atomic steps usually covers whole surface of GaN substrate. The only exception are the layers grown by MOCVD on $(000 \underline{1})_{\mathrm{N}}$ oriented substrates where the hexagonal growth hillocks are often observed. The origin of this morphological features was investigated by Weyher et al. [36].

On the other hand, the growth on the active, $(000 \underline{1})_{\mathrm{N}}$ surface by MBE with both $\mathrm{NH}_{3}$ and plasma $\mathrm{N}$-source results in smooth step flow morphology and almost perfect interfaces between the substrates and deposited layers. Sometimes some light element (probably oxygen) contaminant was found at the interface however it did not disturb the sequence of atomic planes as it was revealed by HREM [28]. It shows that the active surface of $\mathrm{GaN}$ is more sensitive to contamination's which often results in enhanced incorporation of impurities during epitaxy in comparison to the inert surface of the GaN substrates [37].

Probably also due to this reason the best photoluminescence spectra were obtained for layers deposited on the inert, Ga-side of GaN substrates [32, 33]. 
The donor bound exciton lines of the halfwidth below $0.5 \mathrm{meV}$ have been measured on homoepitaxial GaN layers [32]. This confirms the good quality of homoepitaxial layers, resulting in the absence of the strain and extremely narrow excitonic lines.

The GaAlN/GaN multiquantum well (MQW) structures have been obtained using mechano-chemical (0001) surface preparation technique and plasma enhanced MBE growth method [29]. The results of TEM measurements indicate that the dislocation free area of the MQW extends over the range of $100 \mu \mathrm{m}$. [28]. This also indicates on very good crystallographic quality of the substrate surface

\section{CONCLUSIONS}

The results presented in this paper can be summarized by the following points:

1. Two types of GaN single crystals were obtained by HPSG method

a/ conductive - undoped $\mathrm{Ga}$

- max size - $18 \mathrm{~mm}$

- dislocation density $\sim 10^{4} \mathrm{~cm}^{-1}$

b/ SI - Mg doped Ga

- $\max$ size - $14 \mathrm{~mm}$

- dislocation density $\sim 10^{1}-10^{2} \mathrm{~cm}^{-2}$

2. Surface preparation methods were developed

$\mathrm{a} /(0001)_{\mathrm{N}}-$ mechanochemical polishing

$\mathrm{b} /(0001)_{\mathrm{Ga}}$ - reactive ion etching

3. Atomic height step flow growth mode was achieved by MOCVD and MBE methods

4. Near dislocation-free epitaxial layers and MQW structures were grown by both MBE and MOCVD techniques.

\section{ACKNOWLEDGMENT}

The research presented in this paper has been supported by Poland's Committee for Scientific Research grant no 7783495 C/2399. The quantum mechanical calculations have been made using Dmol software distributed by MSI Inc. in the framework of countrywide license supported by Committee for Scientific Research.

\section{REFERENCES}

[1] S. Nakamura, M. Senoh, T. Mukai, Jpn. J. Appl. Phys. 2, Lett. 32, L8 (1993)

[2] S. Nakamura, M. Senoh , N. Iwasa, S. Nagahama, Jpn. J. Appl. Phys. 2, Lett. 34 L797 (1995)

[3] S. Nakamura, M. Senoh, S. Nagahama, N. Iwasa, T. Yamada, T. Matsushita, H. Kiyoku, Y. Sugimoto, Jpn. J. Appl. Phys.2, Lett. 35, L74 (1996)

[4] S. Porowski, Mater. Sci. Eng. B44, 407 (1997)

[5] S. Porowski, I. Grzegory, J. Cryst. Growth 178, 174 (1998)

[6] S. Porowski, I. Grzegory, S. Krukowski, Mater. Res. Soc. Symp. Proc.

[7] J. L. Weyher, S. Müller, I. Grzegory, S. Porowski, J. Cryst. Growth 182, 17 (1997)

[8] J. A. Van Vetchten, Phys. Rev. B7 1479 (1973)

[9] J. Karpinski, J. Jun and S. Porowski, J. Cryst. Growth 66 1(1984)

[10] I. Grzegory, J. Jun, M. Bockowski, S. Krukowski, M. Wroblewski, B. Lucznik and S. Porowski, J. Phys. Chem. Solids 56, 639 (1995)

[11] S. Krukowski, Z. Romanowski, I. Grzegory, S. Porowski, J. Cryst. Growth 189/190, 159 (1998) 
[12] Z. Liliental-Weber, C. Kisielowski, Ruvimov, I. Chen, J. Washburn, I. Grzegory, M. Bockowski, J. Jun, S. Porowski,. J. Electron. Mater. 25 1545(1996)

[13] J-L. Rouviere, J. L. Weyher, M. Seelmann-Eggebert, S. Porowski, Appl. Phys. Lett. 73, 668(1998)

[14] H.P. Maruska, J.J. Tjetjen, Appl. Phys. Lett. 15 (1969) 327

[15] Seifert., W., Franzheld, R., Butter, E., Sobotta, H., Riede V., Cryst. Res. \& Technol. 18 (1983) 383

[16] B. Delley, J. Chem. Phys. 92, 508 (1990)

[17] E. Litwin-Staszewska, to be published

[18] P. Perlin, J. Camassel, W. Knap, T. Talercio, J. C. Chevrin, T. Suski, I. Grzegory and S. Porowski, Appl. Phys. Lett. 67, 2524 (1997)

[19] A. Barcz, T. Suski, unpublished

[20] P. Boguslawski, E. Briggs and J. Bernholz, Phys. Rev. B51, 17255, (1995)

[21] Wook Kim, A. E. Botchkarev, A. Salvador, G. Popovici, H. Tang and H. Morkoc, J. Appl. Phys. 82 (1), 1997

[22] J. Neugebauer and C. G. Van de Walle, Phys. Rev. B50, 8067, (1994)

[23] K. Saarinen, T. Laine, S. Kuisma, P. Hautojarvi, L. Dobrzyñski, J. M. Baranowski, K. Pakuª a, R. Stê pniewski, M. Wojdak, A. Wysmo³ek, T. Suski, M. Leszczyñski, I. Grzegory and S. Porowski, Phys. Rev Lett. 79, 3030, (1997)

[24] S. Porowski, M. Bockowski, B .Lucznik, I. Grzegory, M. Wroblewski, H. Teisseyre, M. Leszczynski, E. Litwin-Staszewska, T .Suski, P. Trautman, K. Pakula and J.M. Baranowski, accepted for Acta Physica Polonica, 1997

[25] T. Suski, O. Ambacher, private communication

[26] I. Grzegory, private communication

[27] M. Leszczynski, I. Grzegory, H. Teisseyre, T. Suski, M. Bockowski, J. Jun, J.M.Baranowski, S. Porowski, J. Domagala, J. Cryst. Growth 169, 235 (1996)

[28] M. Albrecht, private communication

[29] S. Porowski, I. Grzegory, M. Bockowski, M. Leszczynnkki, D Korakakis, A. Bell, I. Harrison, C. T. Foxon, M. Albrecht, H. P. Strunk, J. A. Davidson, P. Dawson, presented in

[30] M. Conway, J. S. Williams and C. Jagadish, private communication

[31] K. Pakula, A. Wysmolek, K. P. Korona, J. M. Baranowski, R. Stepniewski, I. Grzegory, M. Bockowski, J. Jun, S. Krukowski and S. Porowski, Solid State Commun. 97, 919 (1996)

[32] M. Schauler, F. Eberhardt, C. Kirchner, V. Schweigler, M. Kamp, K.J. Ebeling, to be published

[33] P. Beaumont, to be published

[34] A. Zauner, to be published

[35] R. Held, G. Nowak, P. Cohen, S. Porowski, to be published

[36] J. L. Weyher, P. D. Brown, A. Zauner, S. Müller, D. Foord, P. R. Hageman, C. J. Humphreys, P. Larsen, I. Grzegory, S. Porowski, to be published

[37] M. Leszczynski, P. Prystawko, A. Sliwinski, T. Suski, E. Litwin-Staszewska, S. Porowski, Acta Phys. Pol. A 94, 427 (1998) 